\title{
Estudio de impacto de la asimilación de reflectividades radar con HARMONIE-AROME
}

https://doi.org/10.31978/639-19-010-0.309

\author{
Jana Sánchez Arriola ${ }^{1}$ (jsancheza@aemet.es) \\ Beatriz Navascués Fernández-Victorio² (bnavascuesf@aemet.es) \\ Javier Calvo Sánchez 3 (fcalvos@aemet.es) \\ ${ }^{1}$ AEMET / Delegación Territorial en Cantabria \\ ${ }^{2}$ AEMET / Dirección de Planificación, Estrategia y Desarrollo Comercial \\ ${ }^{3}$ AEMET / Dirección de Producción e Infraestructuras / Departamento de Desarrollo y Aplicaciones
}

\begin{abstract}
RESUMEN
La asimilación de datos del modelo HARMONIE-AROME ha mejorado mucho en los últimos meses gracias al esfuerzo de todo el personal del consorcio HIRLAM al que pertenecemos, siendo posible ya introducir en el modelo muy diferentes tipos de observaciones: aviones, satélite, GNSS, radar, etc. Las pasadas operativas del modelo HARMONIE-AROME en AEMET sobre los dominios de la península ibérica y de Canarias se están ejecutando actualmente con una asimilación cada tres horas utilizando observaciones convencionales (procedentes de estaciones en superficie terrestres, de barcos y de boyas, de estaciones de radiosondeos y de aviones), así como radiancias de satélite ATOVS (que suministran información de temperatura y humedad en la vertical), y Zenith Total Delay de satélites GNSS (que contiene información de la columna de humedad y de la presión en superficie). Las observaciones de reflectividad radar, por otro lado, contribuyen a una mejor descripción del campo de humedad del modelo, variable menos observada y que tiene impacto en las predicciones de precipitación, especialmente durante las primeras horas de la integración del modelo.

En este trabajo se presentan los últimos resultados de los experimentos que se han realizado para estudiar el impacto de incorporar también la asimilación de las reflectividades procesadas y difundidas por el proyecto OPERA procedentes de los radares incluidos en los dominios de integración en los que se ejecuta operativamente el modelo HARMONIE-AROME en AEMET.
\end{abstract}

PALABRAS CLAVE: predicción numérica; asimilación de datos; reflectividad; modelización; radares; HARMONIE-AROME.

\section{INTRODUCCIÓN}

La actual pasada operativa de HARMONIE-AROME que se ejecuta en el superordenador Nimbus de AEMET tiene un esquema de asimilación de datos variacional tridimensional (3DVar) con un ciclo de tres horas, y asimila observaciones convencionales (informes de estaciones en superficie SYNOP, de barcos SHIP, de boyas DRIBU, de aviones AMDAR, y de sondeos TEMP), datos de humedad en la columna cenital GNSS ZTD y radiancias de satélite ATOVS de los instrumentos AMSUA, AMSUB y MHS, que proporcionan información en la vertical de temperatura y humedad. La variable menos observada es la humedad, ya que las observaciones que la contienen son únicamente los GNSS ZTD y los ATOVS MHS, cuya frecuencia permite asimilarlos en todos o en una mayoría de ciclos de asimilación, además de los sondeos TEMP que solo están disponibles cada 12 horas. Por otra parte, tanto los datos GNSS ZTD, como las 
radiancias AMSUB y MHS tienen un error sistemático o bias que hay que corregir para que su asimilación produzca un impacto positivo en la predicción. En este sentido, las reflectividades de los radares son una muy buena observación adicional que completa la información de la humedad, tanto por su amplia distribución espacial como temporal, como por su ausencia de bias.

Los datos de la red de radares de AEMET contribuyen en tiempo real al programa OPERA de EUMETNET, donde se procesan, pasan por unos filtros de calidad comunes a todos los de los demás radares de los Servicios Meteorológicos Nacionales europeos, y se armonizan, tras lo cual se elaboran y difunden unos ficheros homogéneos a escala europea que incluyen unos metadatos añadidos, y que los dejan en condiciones de ser asimilados por los modelos numéricos.

En este estudio se han utilizado en concreto los datos de los radares españoles y también portugueses procesados y difundidos por OPERA.

\section{OBSERVACIONES DE HUMEDAD ASIMILADAS EN LA PASADA OPERATIVA HARMONIE-AROME}

Como se ha mencionado, las observaciones de humedad que se están asimilando en la pasada operativa de HARMONIEAROME sobre la península ibérica son las siguientes:

1) Observaciones de GNSS ZTD (retraso cenital total de GNSS). Estos datos son un producto del procesamiento de la señal GNSS de satélite en diferentes centros de proceso en Europa. Tienen una frecuencia temporal muy alta pues se obtienen cada diez minutos, pero la pasada operativa solo asimila una cada tres horas, en concreto la más cercana disponible a la hora del análisis. Además, debido a la correlación espacial de sus errores, la resolución de las que se asimilan se reduce para alcanzar una densidad no mayor de una cada $50 \mathrm{~km}$. Su distribución espacial se muestra en la figura 1.

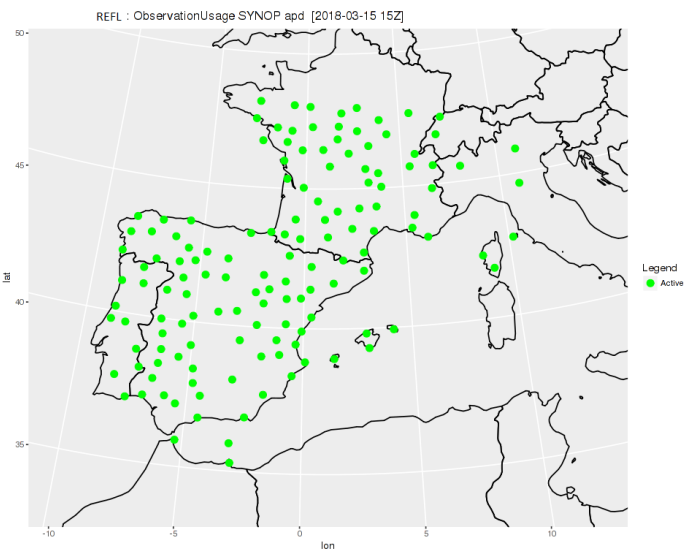

Figura 1. Distribución de las observaciones de GNSS ZTD asimiladas por el experimento REFL el 20180315 a las $15 \mathrm{Z}$.

2) Radiancias ATOVS. Estas observaciones de satélite contienen información sobre la temperatura y la humedad, y aunque su resolución horizontal es muy buena (véase la figura 2) la resolución vertical es bastante pobre. También debido a la correlación espacial de sus errores, antes de asimilarlas se reduce su densidad espacial.

En HARMONIE-AROME se asimilan, relacionados con la humedad, los siguientes canales de los instrumentos AMSUB o MHS (sensores sensibles al contenido de humedad atmosférico):

- los canales 4 y 5 del instrumento MHS del satélite NOAA19, que se asimilan a las $03 \mathrm{Z}$ y $15 \mathrm{Z}$;

- los canales 3, 4 y 5 del instrumento MHS de METOP-A y METOP-B, que se asimilan a las 09Z, $12 \mathrm{Z}$ y $21 \mathrm{Z}$.

Al tratarse de satélites con órbita polar y de dominios geográficos reducidos, no a todas las horas hay observaciones de este tipo disponibles, faltando a las $00 \mathrm{Z}, 06 \mathrm{Z}$ o $18 \mathrm{Z}$ por ejemplo.

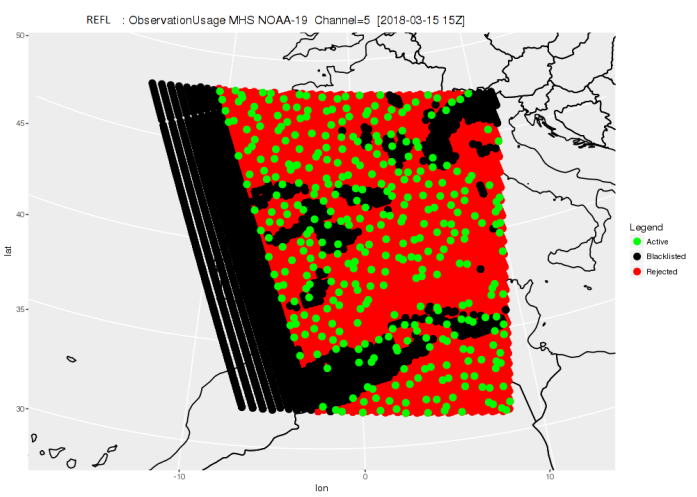

Figura 2. Distribución de las observaciones del canal 5 de ATOVS NOAA19 MHS asimiladas por el experimento REFL el 20180315 a las $15 \mathrm{Z}$. 
3) Sondeos. Como se observa en la figura 3, se dispone de sondeos TEMP a las 00 y las 12 UTC en ocho puntos de España peninsular, Baleares y Canarias, además de en Portugal, Italia, Francia y Suiza, quedando el resto de horas $(03,06,0915,18$ y 21$)$ sin datos de este tipo.

Por otro lado, de todas estas observaciones solo los sondeos no contienen errores sistemáticos, pues las observaciones de satélite GNSS ZTD y ATOVS tienen un bias o sesgo que en HARMONIE-AROME se corrige con un esquema de corrección de bias variacional dentro del proceso de elaboración del análisis cada tres horas. La

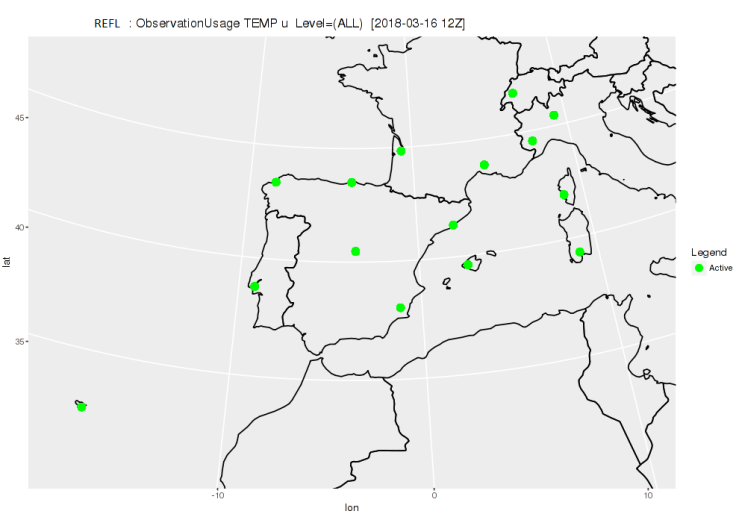

Figura 3. Distribución de las observaciones de TEMP asimiladas por el experimento REFL el 20180316 a las $12 \mathrm{Z}$. humedad de los sondeos, al carecer de bias, se convierte en una «observación de anclaje» a las 00 y las 12 UTC que sirve para identificar y corregir los bias de GNSS ZTD y ATOVS en estos ciclos de asimilación. Se observa por tanto que, en la mayoría de los ciclos de asimilación de la actual configuración de la pasada operativa, existe un déficit de información de humedad para poder construir el estado inicial del modelo, y por otro lado hay una falta de observaciones de «anclaje» para el esquema de corrección de bias de las observaciones de satélite.

Los ficheros de observaciones de radar de OPERA que se reciben en AEMET contienen datos filtrados de reflectividad y de vientos Doppler. Este filtrado inicial es un preproceso que se realiza en OPERA con los datos brutos de los radares de todos los países que lo integran. Por otro lado, se introducen además en los ficheros algunos «metadatos» que son útiles para su posterior asimilación por parte de los modelos numéricos, como por ejemplo los resultados de los chequeos de calidad a los que estas observaciones han sido sometidas. Aunque están disponibles datos de todos los radares portugueses, españoles y franceses, en este estudio se han utilizado solamente los dos primeros, dejando la asimilación de los franceses para estudios posteriores. En un principio solo se ha utilizado la variable de reflectividad.

El código de referencia del ciclo 40 del modelo HARMONIE-AROME está ya preparado para asimilar tanto la reflectividad como el viento Doppler de radares a través de ficheros de OPERA, aunque ha habido que hacer una serie de cambios a nivel local para adaptarlo al superordenador Nimbus y para la implementación de este sistema en AEMET. En el caso de la reflectividad radar, solo se seleccionan las observaciones con elevaciones mayores de 1 grado, y de aquellas en las que el flag de calidad asignado en el preproceso de OPERA supera un determinado umbral, se realiza una reducción de su resolución para evitar los efectos de los errores de las observaciones correlacionados espacialmente, para lo cual se generan unas superobservaciones según ciertos criterios (RIDAL et al., 2017).

La relación entre las variables del modelo y las reflectividades es no lineal y es por tanto compleja, ya que tiene en cuenta parametrizaciones de procesos de microfísica. Por otro lado, los errores de reflectividad muestran distribuciones no gaussianas. Por estos motivos, en el sistema HARMONIE la variable reflectividad no se asimila directamente en el modelo sino que previamente se procesa y se transforma en un perfil vertical 1D de humedad relativa (RH) como se describe en CAUMONT et al. (2010). En este algoritmo, la reflectividad se transforma en un perfil vertical de humedad en un procedimiento que incluye la comparación entre la reflectividad simulada por el modelo y la observada. Los perfiles de humedad que se asimilan contribuyen entonces a humedecer o secar los campos de humedad del first guess o campo previo del modelo. Además, hay que tener en cuenta que este first guess se construye en cada ciclo de asimilación mediante un proceso de combinación o blending de una predicción a 3 horas de HARMONIE con los campos previstos del ECMWF de menor alcance disponibles que verifican a la hora del análisis. Ello hace que la influencia de las observaciones asimiladas en posteriores ciclos de asimilación se reduzca sustancialmente. 


\section{EXPERIMENTOS DE IMPACTO DE LA ASIMILACIÓN DE REFLECTIVIDAD RADAR EN HARMONIE-AROME}

Para estudiar el impacto de la asimilación de reflectividad radar en el modelo HARMONIE-AROME sobre el dominio de la península ibérica ha sido necesario lanzar varios experimentos. El primero se ha ejecutado para un periodo previo que se llama de spin-up con objeto de calibrar los coeficientes de bias de las observaciones de GNSS ZTD en presencia de los nuevos datos de humedad procedentes de las reflectividades radar. Posteriormente, se han ejecutado los dos experimentos para analizar el impacto de las reflectividades radar durante el periodo de estudio: uno de control que es exactamente igual que la actual pasada operativa, y el otro paralelo a control pero añadiendo la asimilación de la reflectividad de los radares españoles y portugueses.

\subsection{Periodo de spin-up}

Para calibrar los coeficientes del bias de las observaciones GNSS ZTD para este estudio, se ha ejecutado un experimento paralelo a control (con observaciones convencionales + GNSS ZTD + ATOVS) sobre la península ibérica pero asimilando los GNSS ZTD de forma pasiva (es decir, sin influir en el análisis) y adicionalmente la reflectividad radar de forma activa. Este experimento se ha llevado a cabo para un periodo de 16 días, del 1 al 16 de febrero de 2018. La figura 4 muestra la evolución de los coeficientes del bias de las observaciones GNSS ZTD de dos estaciones en este experimento para el periodo de spin-up. Se observa cómo se adaptan progresivamente con el transcurso del tiempo, y cómo a partir de un ciclo de asimilación (eje x) equivalente a 15 días el valor del bias se empieza a estabilizar.
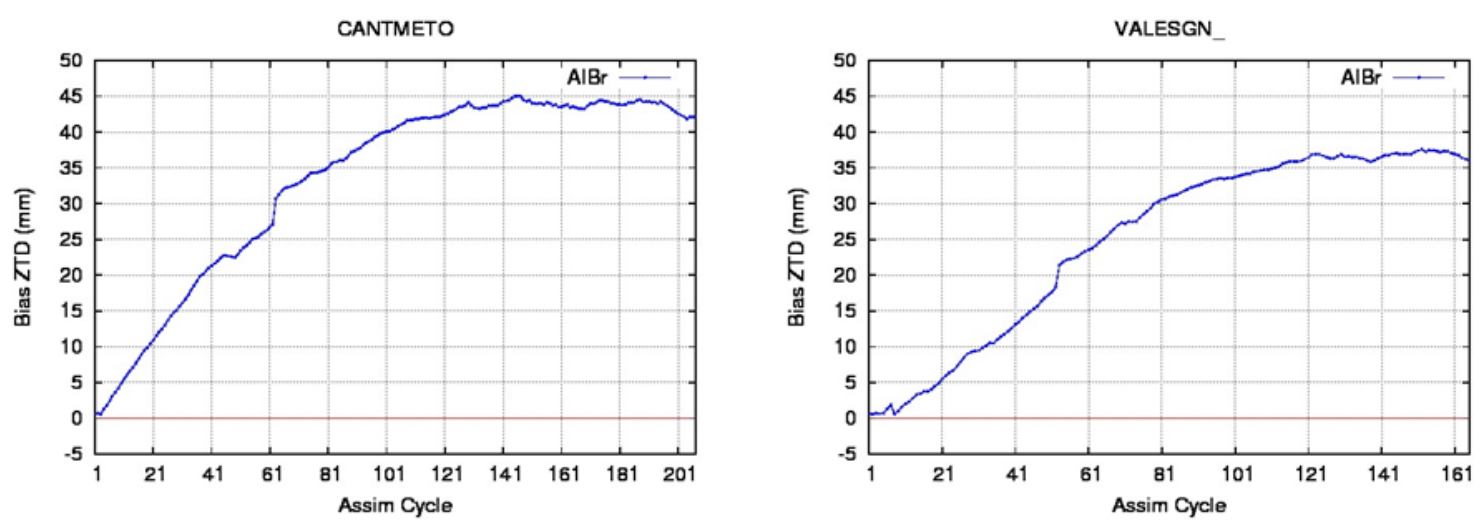

Figura 4. Evolución de los coeficientes bias de las observaciones GNSS ZTD de las estaciones CANTMETO y VALESGN_ del experimento de spin-up.

\subsection{Periodo de estudio}

Este periodo comprende del 16 de febrero al 31 de marzo 2018. Una vez calibrados los coeficientes de bias de los GNSS ZTD, se empieza el periodo de estudio con la versión 40h1.1 de HARMONIE-AROME, usando 3DVar con ciclo de 3 horas en dos experimentos:

1) CONTROL: asimilando observaciones convencionales, ATOVS y GNSS ZTD activos. Este experimento es idéntico a la pasada operativa.

2) REFL: a las observaciones asimiladas en CONTROL se añaden las reflectividades radar de ficheros de OPERA de los radares de España y Portugal.

Y serán estos dos experimentos los que se compararán en estas seis semanas de estudio. 


\section{MONITORING DE LA ASIMILACIÓN}

Para comprobar que el modelo se está comportando de forma coherente al introducir las observaciones de reflectividad, primero se analiza si el cambio que hace el análisis debido a estas está de acuerdo con el resto de observaciones de humedad que tenemos. Para ello, se comprueba que la distancia entre las observaciones y el análisis es menor que la de las observaciones y el first guess para el conjunto de observaciones asimiladas con información de humedad: GNSS ZTD, los canales de MHS, la humedad especifica $q$ de los radiosondeos, y los perfiles de humedad relativa derivados de los perfiles de reflectividad. Se observa que en el caso de la humedad relativa de radar a $800 \mathrm{hPa}$ (figura 5) efectivamente esta distancia disminuye (el análisis se acerca más a las observaciones), y también en el caso de los GNSS ZTD (figura 6), en el del canal 5 MHS METOP-A de satélite (figura 7) y en la humedad específica $q$ de los radiosondeos a $800 \mathrm{hPa}$ (figura 8). Todo ello indica que efectivamente el cambio producido por el análisis debido a la asimilación de estas nuevas observaciones es coherente con el causado por el resto. En general, se aprecia que las observaciones secan un modelo que parece que está demasiado húmedo, lo cual, según veremos en el apartado siguiente, se traducirá en un resultado positivo en la habilidad de predicción en los campos ligados a la humedad.

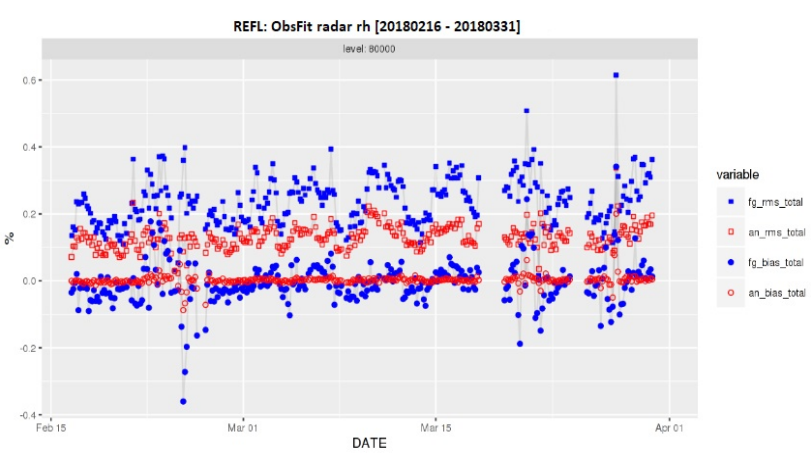

Figura 5. Evolución temporal del error cuadrático medio (ECM) y bias del first guess departure (ob-fg) en azul y analysis departure (ob-an) en rojo de la HR de radar a $800 \mathrm{hPa}$ del experimento con reflectividad radar.

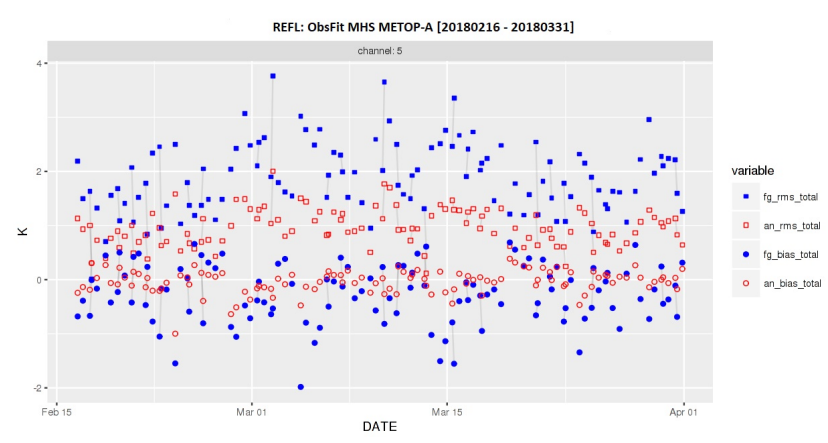

Figura 7. Como la figura 5 para el canal 5 MHS del satélite METOP-A.

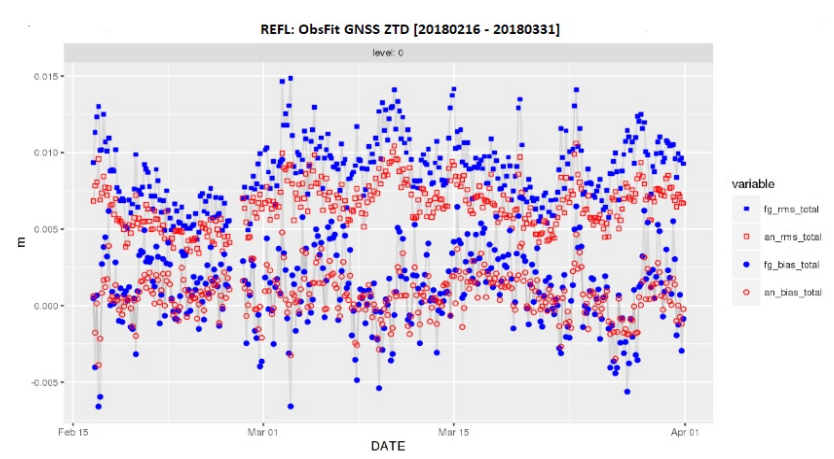

Figura 6. Como la figura 5 para los GNSS.

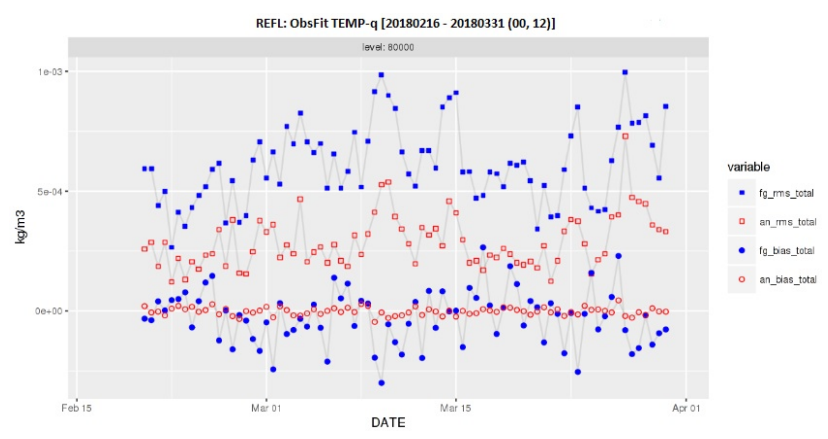

Figura 8. Como la figura 5 para la humedad específica $q$ de los sondeos a $800 \mathrm{hPa}$.

Por otro lado, si comparamos los valores del ECM de las distancias entre las observaciones y el campo previo (ob-fg) y de la distancia entre las observaciones y el análisis (ob-an) del experimento de control CTRL y el de reflectividad REFL se observa (figura 9) que la diferencia entre ambos es muy pequeña, como cabía esperar debido a la inclusión de los campos previstos más recientes del modelo del ECMWF disponibles en el análisis de cada ciclo (blending) que, como se ha comentado anteriormente, hace que el efecto progresivo que una observación pueda hacer en el campo previo o first guess quede suavizado. 


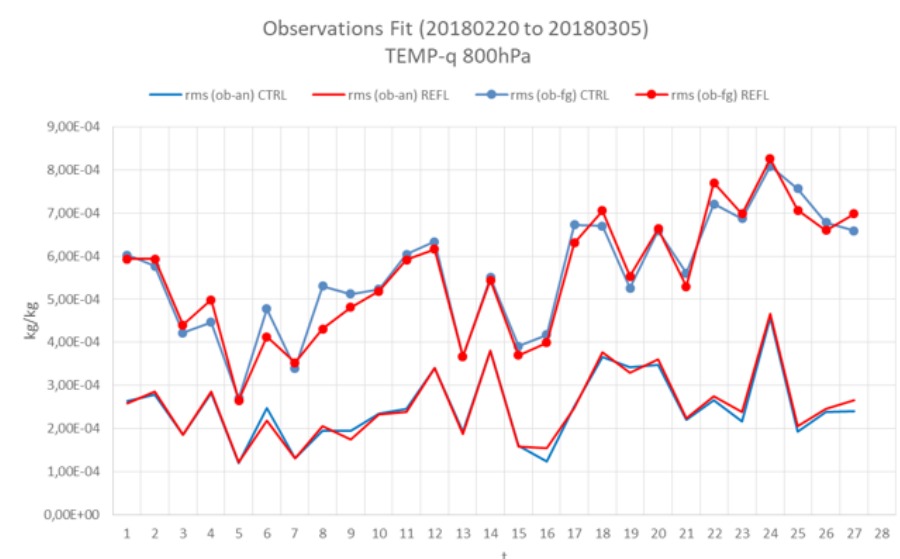

Figura 9.

Evolución temporal del ECM del $F G$ departure (línea con puntos) y del $A N$ departure (línea lisa) de la humedad específica $q$ de los sondeos a $800 \mathrm{hPa}$ de los experimentos CTRL (azul) y REFL (rojo) para un periodo de dos semanas.

\section{RESULTADOS: VERIFICACIÓN DE LAS PREDICCIONES}

La evaluación de los experimentos en este periodo de seis semanas se ha realizado mediante dos tipos de verificación: una objetiva de todo el periodo, y otra subjetiva de algún caso de estudio que hubo en estos días.

\subsection{Verificación objetiva}

El impacto general de la asimilación de las reflectividades de radar en la península ibérica en el periodo estudiado es neutro en las variables no relacionadas con la humedad, y de neutro a positivo en las que sí están relacionadas con la humedad como son la propia humedad relativa, la temperatura de termómetro húmedo y sobre todo en la precipitación. Este impacto se observa fundamentalmente en el corto alcance: H+03 y H+06.

En la figura 10 se muestra la diferencia de los errores del experimento de control CTRL y el que asimila reflectividad REFL relativa al error de CTRL para la precipitación acumulada en $3 \mathrm{~h}$ para todo el periodo. Se puede observar que la asimilación de reflectividades produce una mejora significativa de la precipitación en los primeros alcances (hasta $\mathrm{H}+6$ ), y además hay que remarcar que este resultado es estadísticamente significativo con una confianza del $90 \%$. Para H+09 también encontramos mejora pero esta ya no es estadísticamente significativa.

Este resultado se debe sobre todo a la reducción de «falsas alarmas» en el caso de asimilar la reflectividad como se puede ver en la figura 11.

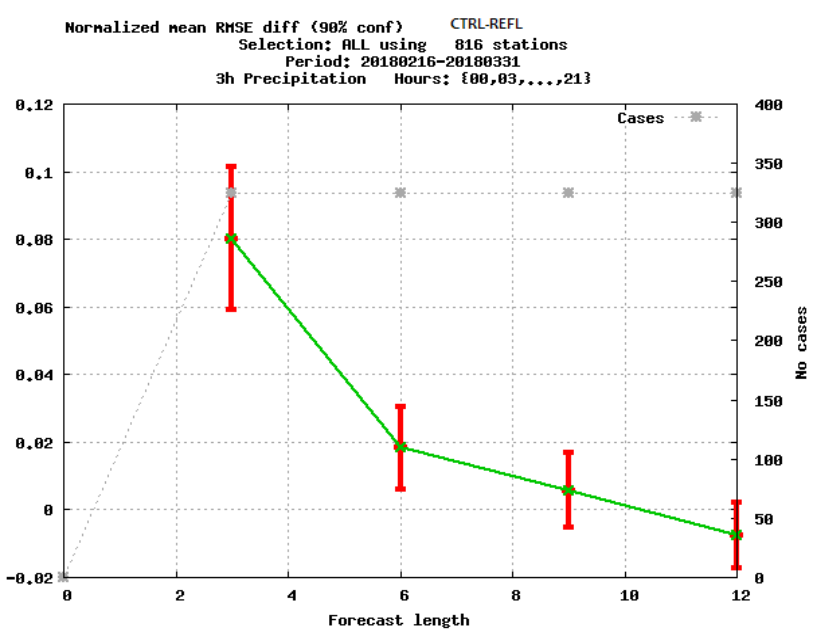

Figura 10. Diferencia de errores de CTRL y REFL en función del alcance de predicción hasta 12 horas después del análisis. $\left(\mathrm{RMSE}_{\mathrm{CTRL}}-\mathrm{RMSE}_{\mathrm{REFL}}\right) / \mathrm{RMSE}_{\mathrm{CTRL}}$.

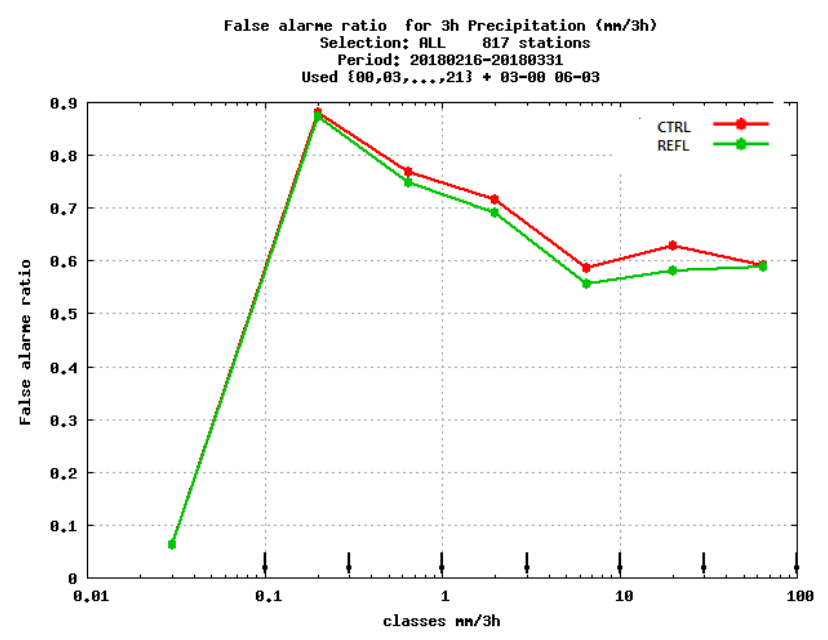

Figura 11. Tasa de «falsas alarmas» de ambos experimentos: línea roja CTRL y verde REFL. 

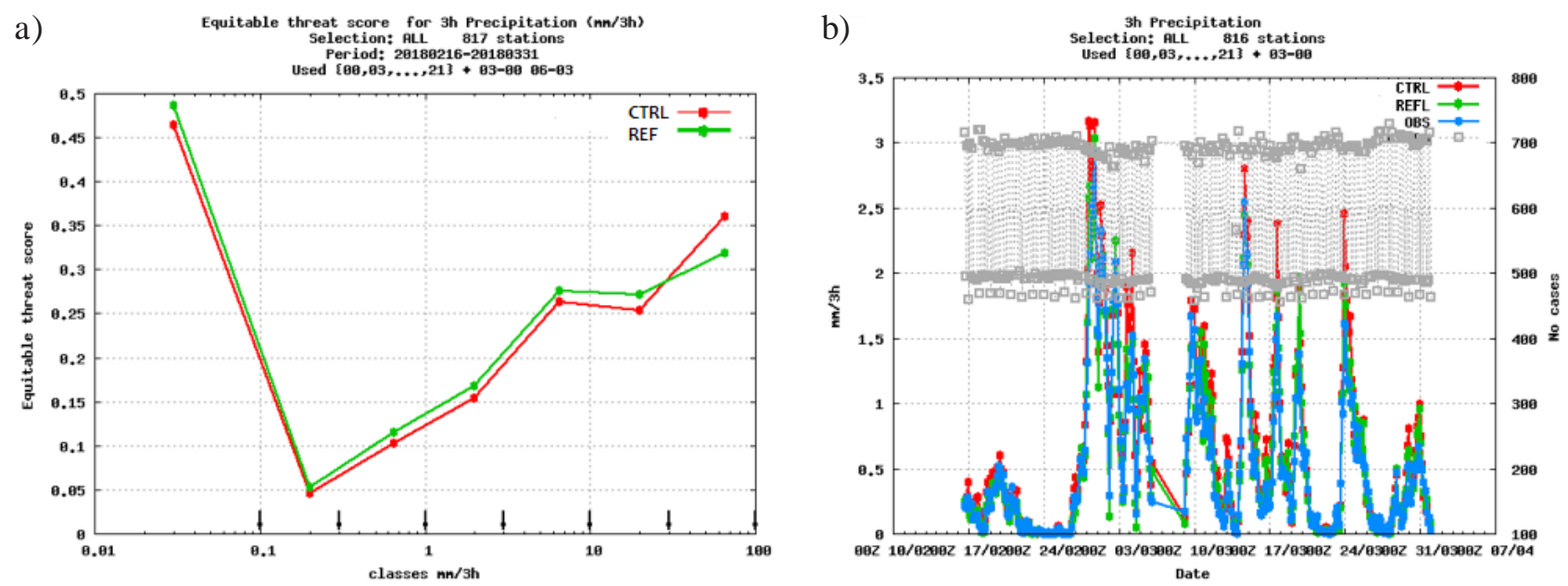

Figura 12. Para los experimentos de control, CTRL (línea roja) y con asimilación radar (línea verde),

a) Equitable Thread Score (ETS), en el que valores más altos indican mejor predicción y

b) evolución temporal de la precipitación media de ambos experimentos y de las observaciones, estas en azul.

En la figura 12a se aprecia que el experimento que asimila reflectividad tiene mayor habilidad para predecir la precipitación que el control (mayor ETS) mientras que el experimento de control tiende a sobreestimar más los valores medios de la precipitación (figura 12b).

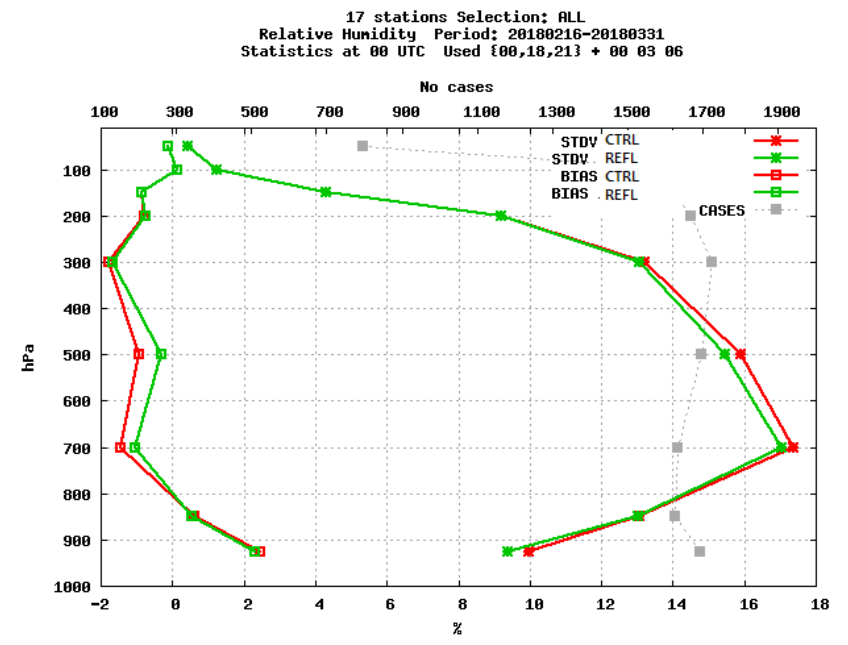

Por otro lado, la figura 13 muestra como la humedad relativa también mejora en el experimento que asimila reflectividad radar.

Figura 13.

Perfil vertical de los errores (BIAS y STDV) de la humedad relativa para los experimentos de control, CTRL (línea roja) y con asimilación radar (línea verde) frente a las observaciones de sondeos TEMP, a las $00 \mathrm{Z}$.

\subsection{Verificación subjetiva: caso de estudio}

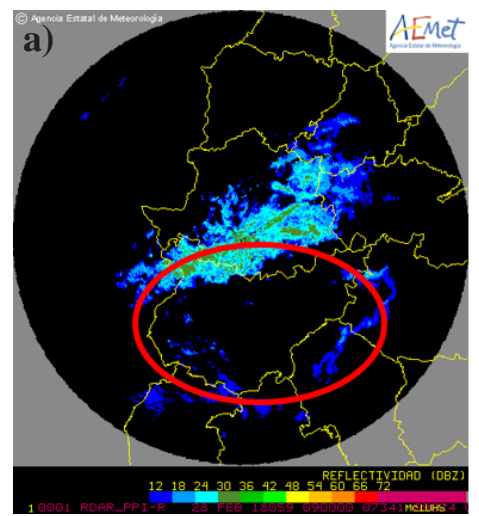

$\begin{array}{cc}\text { CTRL Reflectividad } 1000 \mathrm{~m}(\mathrm{dBZ}) \\ 28 / 02 / 201806 z & \mathrm{H}+03 \text { Valid: } 28 / 02 / 201809 \mathrm{z}\end{array}$

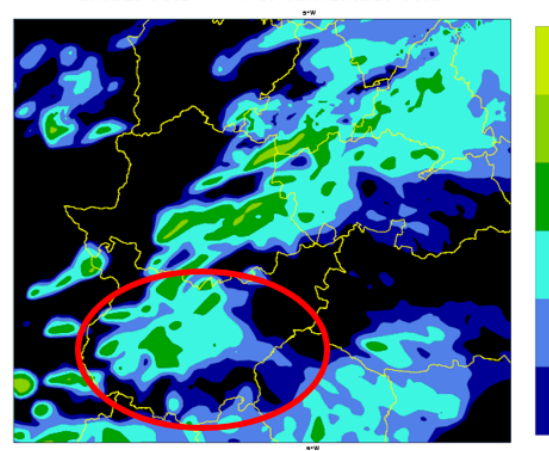

$\begin{array}{ll}\text { REFL } & \text { Reflectividad 1000m (dBZ) } \\ \text { C) } & \text { H+03 Valid: } 28 / 02 / 201809 z\end{array}$

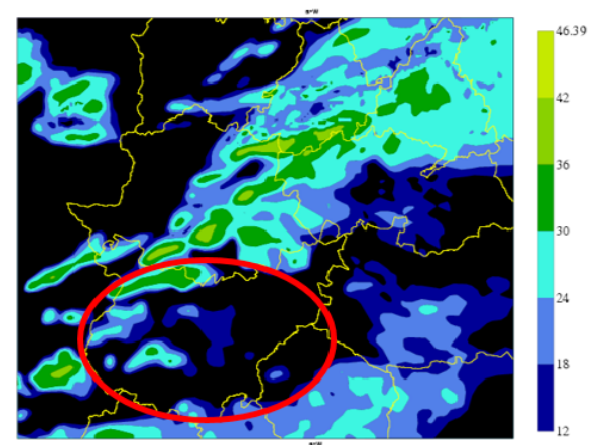

Figura 14. Caso de estudio correspondiente al día 28 de febrero a las 09Z. a) Imagen de reflectividad observada por el radar, b) imagen simulada por el experimento CTRL y c) imagen simulada por el experimento REFL. Los diferentes colores son la reflectividad en dBZ. 
Si comparamos imágenes de reflectividad simuladas por las dos versiones del modelo, encontramos que las diferencias en general no son muy grandes aunque sí que parecen confirmarse los resultados de la verificación objetiva, tendiendo el experimento de control cuyo estado inicial es más húmedo, a producir más precipitación y generar más falsas alarmas como puede verse en el ejemplo de la figura 14.

\section{CONCLUSIONES Y FUTURAS LÍNEAS DE TRABAJO}

El impacto de la asimilación de reflectividad radar en HARMONIE-AROME operativo se ha estudiado para un periodo de seis semanas entre febrero y marzo de 2018. Se ha obtenido un pequeño impacto positivo en la precipitación, ya que en general se ha disminuido la tasa de «falsas alarmas». La mejora se produce solo en los primeros alcances de predicción (hasta unas $9 \mathrm{~h}$ ), pudiendo ser este resultado relevante para una pasada de HARMONIE-AROME en apoyo del nowcasting.

Se ha comprobado con este estudio que la asimilación de reflectividad radar de OPERA permite tener un conocimiento más realista de la humedad observada en nuestro modelo, lo que se traduce a un mejor análisis, lo cual además de implicar una mejora en la habilidad de predicción (precipitación y humedad), ayuda a calibrar mejor los coeficientes de bias de las variables de satélite relacionadas con la humedad.

Mencionar que si bien este artículo solo trata del dominio de la península ibérica, en el dominio de Canarias también se han hecho estudios de impacto con el único radar (Las Palmas) que hay disponible, y asimismo se ha obtenido un impacto ligeramente positivo, aunque tendrá que ser mejor estudiado.

Como futuras líneas de trabajo, en una ampliación de este estudio, se van a incluir los radares franceses pues son bastante numerosos y muchos de ellos entran en el dominio de nuestra pasada operativa. Por otro lado, se estudiará la combinación de las reflectividades con los vientos Doppler, también disponibles en estos ficheros de OPERA.

Otras mejoras previstas en la asimilación de datos de HARMONIE-AROME en AEMET serán la asimilación de vientos de escaterómetros, de humedad procedente de aviones, de humedad procedente de la observación de satélite SEVIRI y de las observaciones de satélite IASI.

\section{AGRADECIMIENTOS}

Queremos agradecer la ayuda de Martin Ridal del SMHI en la fase de preproceso y asimilación de estas observaciones en el Cy40h1.1, y en la fase de instalación y adecuación de los experimentos al superordenador Nimbus a Daniel Martín Pérez de Numérica y a Ángel Álvarez García-Botija de Sistemas.

\section{REFERENCIAS}

BENGTSSON, L. et al., 2017. The HARMONIE-AROME Model Configuration in the ALADIN-HIRLAM NWP System. Monthly Weather Review, 145, 5, 1919-1935.

Caumont, O., Ducroce, V., Wattrelot, E., Jaubert, G. y Pradier-Vabre, S., 2010. 1D+3DVar assimilation of radar reflectivity data: a proof of concept. Tellus A: Dynamic Meteorology and Oceanography, 62:2, 173-187.

Ridal, M. y Dahlbom, M., 2017. Assimilation of Multinational Radar Reflectivity Data in a Mesoscale Model: A Proof of Concept. Journal of Applied Meteorology and Climatology, 56 (6), 1739-1751. 